Original article

\title{
Influence of different dialysis modalities in the measurement of resting energy expenditure in patients with acute kidney injury in ICU
}

\author{
Cassiana R. de Góes*, Barbara Perez Vogt, Ana Claudia S. Sanches, André L. Balbi, \\ Daniela Ponce
}

Faculdade de Medicina de Botucatu, UNESP Univ Estadual Paulista, Department of Internal Medicine, Botucatu, São Paulo, Brazil

\section{A R T I C L E I N F O}

\section{Article history:}

Received 10 May 2016

Accepted 11 August 2016

\section{Keywords:}

Acute kidney injury

Dialisys

Energy expenditure

Indirect calorimetry

Intensive care

\begin{abstract}
S U M M A R Y
Background: Currently, the execution of indirect calorimetry, which is considered the gold standard for measuring energy expenditure, is not indicate during dialysis, and it may interfere on nutritional therapy of these patients. This study aimed to evaluate the resting energy expenditure (REE) in patients with severe acute kidney injury treated by different modalities of dialysis and to identify whether dialysis influences on REE.

Methods: This was a prospective cohort study that evaluated patients admitted in intensive care units with diagnosis of acute kidney injury AKIN-3, mechanically ventilated, and submitted to conventional hemodialysis (CHD), extended hemodialysis (EHD) or high volume peritoneal dialysis (HVPD). Indirect calorimetry was performed at pre dialysis time and during the dialysis procedure. Parameters that could change REE were also evaluated.

Results: One-hundred patients undergoing 290 dialysis sessions were evaluated, with mean age $60.3 \pm 17$ years, $69 \%$ were male and $74 \%$ have died. There was no significant difference between REE of predialysis time and during dialysis time ( $2156 \pm 659 \mathrm{kcal}$ vs. $2100 \pm 634 \mathrm{kcal}$, respectively, $\mathrm{p}=0.15)$. No difference was observed in the REE before and during dialysis of different modalities. There were no differences between parameters pre and during dialysis of each modality. There was only a difference in norepinephrine dose, which was higher in pre dialysis time in HVPD and EHD modalities, compared with CHD modality. Moreover, during dialysis time, EHD modality had significantly higher VAD compared to other dialysis modalities.

Conclusion: The three evaluated modalities did not change REE. Indirect calorimetry can be performed during dialysis procedures and there was no difference between ventilation parameters, sedatives use, body temperature and VAD in both moments.
\end{abstract}

(C) 2016 Elsevier Ltd and European Society for Clinical Nutrition and Metabolism. All rights reserved.

\section{Introduction}

Acute kidney injury (AKI) is a common complication in hospitalized patients and affects approximately $35 \%$ of patients in intensive care unit (ICU) [1]. Severe AKI requiring dialysis is a condition associated with high morbidity and mortality [2]. Observational studies have reported an association between malnutrition, caloric deficit, and worse renal outcome and patients survival in ICU $[3,4]$. The condition of these patients is complex because of changes in use of substrates, insulin resistance,

\footnotetext{
* Corresponding author. Paranapanema Avenue, 165, Avaré, São Paulo 18701-240, Brazil. Fax: +55 1438116005.
}

E-mail address: cassiana.goes@yahoo.com.br (C.R. Góes). hyperglycaemia, and hypercatabolism in response to neuroendocrine alterations and inflammatory mediators. Such complex physiological disorders turn the nutritional therapy more difficult [5,6].

Adjusting nutritional support of these patients is important to prevent complications associated with over and underfeeding, which increase morbidity and mortality rates. The accurate determination of caloric requirement is necessary since it is specific to each person, varying according to age, weight, height, race and it is also affected by primary disease, treatments and evolution [7]. Energy supply goal estimated by indirect calorimetry (IC) is recommended by guidelines and it has associated with better outcomes in critically ill patients $[3,8]$.

Although considered the gold standard for energy expenditure (EE) measurement in critically ill patients, IC has limitations in 
clinical practice in ICU. High cost and the need for specialized staff can be limiting factors in several centers. Moreover, many patients are ineligible for this measurement according to the recommendations. The reasons for ineligibility may be high fractions of inspired oxygen $\left(\mathrm{FIO}_{2}\right)$, air leakage on the system or originated from chest tubes; patient hemodynamic instability; recent changes or disconnect in ventilator and extracorporeal circulation, such as oxygenation or hemodialysis [9-11].

When AKI is severe, dialysis is often required as long as treatments for specific conditions are applied. AKI requiring renal replacement therapy (RRT) prevalence has increased in recent years [12,13]. Two modalities of dialysis are usually provided: continuous or intermittent. Both methods achieve a satisfactory degree of metabolic control, but require hours of therapy. There are also recommendations to not perform IC during dialysis may preclude EE measurement and hinder nutritional therapy in these patients.

Patients' ineligibility to IC is supported by older studies which showed the increase of oxygen uptake (VO2) and removal of $\mathrm{CO}_{2}$ during hemodialysis. Both may alter the EE measurement [14,15], but this contraindication needs to be further investigated, due to measuring EE importance in AKI patients.

Thus, the present study aims to evaluate the resting energy expenditure (REE) measured by IC of severe AKI patients undergoing different dialysis modalities, and identify if dialysis influences REE.

\section{Subjects and methods}

Prospective cohort study that evaluated patients older than 18 years admitted, from March 2013 to December 2015, in ICU with diagnosis of AKI stage 3 according to the criteria of KDIGO 2012 [16], with clinical diagnosis of acute tubular necrosis (ATN), and necessity for renal replacement therapy were included. Patients were mechanically ventilated and IC was performed daily, prior to dialysis (non-dialysis time), and during dialysis procedure.

Patients with AKI caused by other etiologies stages 4 and 5 chronic kidney disease (Glomerular Filtration Rate - TFG - <30 ml/ min estimated by the Modification of Diet in Renal Disease MDRD) [17] were excluded. To estimate TFG, patient's baseline serum creatinine, defined as the most recent serum creatinine value obtained before admission, not preceding 12 months of the hospitalization date was considered. If this value was unknown or from 12 months before admission, the lowest value of baseline serum creatinine observed during follow-up was considered [18]. Kidney transplant patients were also excluded.

Patients with factors that could lead to inaccuracy in the REE measurement by IC were also excluded. These factors can be: fraction of inspired oxygen $\left(\mathrm{FiO}_{2}\right)$ greater than $60 \%$; positive endexpiratory pressure (PEEP)> $10 \mathrm{~cm} \mathrm{H}_{2} \mathrm{O}$; presence of agitation; use of neuromuscular blockers and any leakage of air (into the ventilator circuit, around the endotracheal tube cuff, or inpatient from a drain or bronchopleural fistula).

The protocol was initiated at the time of dialysis indication, and terminated at the time of the dialysis suspension due to recovery of renal function or death.

This study was approved by the institution ethics committee. The consent form was signed by the participant's legal guardian prior to entry into the study.

\subsection{Energy expenditure measure}

REE is usually measured due to the impossibility of achieving the conditions for measuring the basal EE in critical patients. To ensure REE measurement, the patients were in supine position resting for at least 30 min prior to the measurement. The environment was thermoneutral for the $30 \mathrm{~min}$ prior to the measurement and during it. They have not used additional painkillers and sedatives within $30 \mathrm{~min}$ of IC initiation; no procedures within 60 min before the IC beginning (only the dialysis procedure, during dialysis time); no general anesthesia within $8 \mathrm{~h}$ of IC onset; and continued parenteral and/or enteral nutrition during the data collection period.

IC was performed using calorimeter Quark RMR unit (Cosmed, Rome, Italy), which was calibrated before each measurement. The exam lasted $30 \mathrm{~min}$ on average. It was desired that patients reached steady state during the test. The steady state was defined as a variation $<10 \%$ in the oxygen consumption measurements (VO2) and carbon dioxide production ( $\mathrm{VCO} 2$ ), and $<5 \%$ in respiratory quotient in every minute.

In addition to REE measured in two moments, parameters that can influence REE were also evaluated, as ventilatory parameters (minute volume, total current volume, PEEP, FIO2), continue sedation and vasoactive drugs during all measurements.

\subsection{Dialysis}

The dialysis method was chosen by nephrologists, according to patient's clinical condition, namely:

- High volume peritoneal dialysis (HVPD): Sessions conducted through flexible catheter implanted in peritoneal cavity ("Tenckhoff" catheter), performed at bedside and with no direct visualization by the nephrology team. The dialysis solution used was Dianeal Baxter $(\mathrm{Na}=135 \mathrm{mEq} / \mathrm{L}, \mathrm{Ca}=3.5 \mathrm{mEq} / \mathrm{G}$, $\mathrm{K}=0 \mathrm{mEq} / \mathrm{L}, \mathrm{Mg}=1.5 \mathrm{mEq} / \mathrm{L}$, lactate $=40 \mathrm{mEq} / \mathrm{L}$, Glucose $=1.5$, 2. 5 or $4.25 \%$ ), with exchanges done by HomeChoice cycler (Baxter). Continuous sessions were performed, each session lasting 1 day (24 h).

- Conventional Hemodialysis (CHD): Sessions were conducted through double lumen catheter in central venous access (jugular, subclavian or femoral), using machine Fresenius $4008 \mathrm{~S}$ or Gambro K200S and capillary cellulose acetate or polysulfone. Each session lasted $4 \mathrm{~h}$, using blood flow 200-300 ml/min and dialysate flow 300-500 ml/min. The dialysis bath concentrations were adjusted according to patient individual necessity.

- Extended Hemodialysis (EHD): Sessions conducted through double lumen catheter inserted into a central venous access (jugular, subclavian or femoral), using machine Fresenius $4008 \mathrm{~S}$ or Gambro K200S and capillary cellulose acetate or polysulfone. Each session lasted 6 or $10 \mathrm{~h}$, blood flow 200-300 ml/min and dialysate flow $300-500 \mathrm{ml} / \mathrm{min}$. Dialysis bath concentrations were adjusted according to patient individual necessity.

\subsection{Statistical analysis}

Results are presented as median and first and third quartiles, mean \pm standard deviation or percentage, according to variables distribution.

For comparisons of clinical characteristics of patients among different dialysis modalities, ANOVA with post hoc Tukey, or Kruskal-Wallis test with post hoc Dunn was used. The analysis of the moments before and during dialysis in each dialysis modality was performed using the mixed model for repeated measures followed by Tukey for variables with symmetrical distribution or generalized linear model with gamma distribution for variables with asymmetric distribution. Categorical variables were analyzed by trend test to check changes between the moments. It was adopted as statistically significant $\mathrm{p}<0.05$. 


\section{Results}

One-hundred twenty-three AKI patients at the time indication of the dialysis procedure were evaluated. Of these, 23 patients were excluded because the measurement during dialysis time was precluded, due to instability and agitation. Figure 1 shows the patients characterization.

Regarding to clinical characteristics, age average was $60.3 \pm 17$ years and $69 \%$ were male. Etiology of AKI was associated with sepsis in most patients (80\%). Specific prognostic index for ATN (ATN-ISS) average was $0.65 \pm 0.17$. Table 1 shows clinical characteristics of general study population.

Eighty-three patients were treated by HD and 17 patients by PD. Groups treated with CHD and EHD were similar in age $(57 \pm 18.2$ years and $60 \pm 16.8$ years, respectively, $\mathrm{p}>0.05)$ and the group treated by HVPD was older ( $69 \pm 12.5$ years, $\mathrm{p}=0.03$ compared to patients treated with $\mathrm{CHD}$ ). Regarding gender, there is male predominance in all modalities (72.5\% for CHD, 72\% for EHD and 53\% for HVPD). Patients of the three dialysis modalities were similar regarding specific prognostic index for ATN (ATN-ISS) $(0.64 \pm 0.2$ to HVPD, $0.62 \pm 0.18$ to CHD and $0.67 \pm 0.15$ in EHD; $p>0.05$ ), etiology of AKI (predominance of the etiology associated with sepsis in all modalities), and outcome. However, there was difference in primary diagnosis among patients treated by PD and HD (prevalence of cardiovascular disease in patients treated by HVPD, while there was predominance of infectious diseases in patients undergoing hemodialysis). Table 2 shows clinical characteristics of patients undergoing different dialysis modalities.

Analyzing all the patients included in this study, significant difference between REE of predialysis time and during dialysis time was not found $(2156 \pm 659 \mathrm{kcal}$ vs. $2100 \pm 634 \mathrm{kcal}$, respectively, $\mathrm{p}=0.15$ ), as shown in Table 1 . When times before and during dialysis of different modalities were compared, no difference was observed in the REE, as shown in Table 3.

To assess parameters that may alter REE in patients treated by different dialysis modalities, some variables, as ventilatory parameters, sedation, vasoactive drug dose (VAD), and body
Table 1

Clinical characteristic of general study population.

\begin{tabular}{ll}
\hline Parameters & \\
\hline Age (years) & $60.3 \pm 17$ \\
Male (\%) & $69(69)$ \\
AKI etiology (\%) & \\
Associated with sepsis & $80(80)$ \\
Ischemic & $12(12)$ \\
Nephrotoxic & $6(6)$ \\
Mixed & $2(2)$ \\
ATN-ISS & $0,65 \pm 0,17$ \\
REE pre dialysis time (Kcal) & $2156 \pm 659$ \\
REE during dialysis (Kcal) & $2100 \pm 634$ \\
Weight (kg) & $77.8 \pm 21.7$ \\
Primary diagnosis (\%): & \\
Cardiovascular disease & $33(33)$ \\
Sepsis, severe sepsis, shock & $46(46)$ \\
Cancer & $8(8)$ \\
Liver diseases & $7(7)$ \\
Trauma & $6(6)$ \\
Outcome (\%) & \\
Recovery of renal function & $18(18)$ \\
Chronic dialysis & $8(8)$ \\
Death & $74(74)$ \\
\hline
\end{tabular}

AKI - Acute kidney Injury, ATN-ISS - Acute Tubular Necrosis individual Score Severity, REE - Resting Energy Expenditure.

temperature were analyzed. The results are shown in Table 3. Significant differences between those parameters pre and during dialysis of each modality were not observed.

Comparing different modalities, there was a difference in norepinephrine dose (VAD), which in the pre dialysis time was significantly higher in HVPD and EHD modalities, compared with CHD modality $[0.23(0.18-0.82) \mathrm{mcg} / \mathrm{kg} / \mathrm{min} ; 0.54(0.4-0.92) \mathrm{mcg} /$ $\mathrm{kg} / \mathrm{min}$ and $0.03(0-0.12) \mathrm{mcg} / \mathrm{kg} / \mathrm{min}$, respectively, $\mathrm{p}<0.001]$. Moreover, during dialysis time, EHD modality had significantly higher VAD compared to other dialysis modalities [0.68 (0,4-1) $\mathrm{mcg} / \mathrm{kg} / \mathrm{min}$ in EHD; $0.13(0.04-0.75) \mathrm{mcg} / \mathrm{kg} / \mathrm{min}$ in HVPD and $0.03(0-0.15) \mathrm{mcg} / \mathrm{kg} / \mathrm{min}$ in the CHD, $\mathrm{p}<0.001]$.

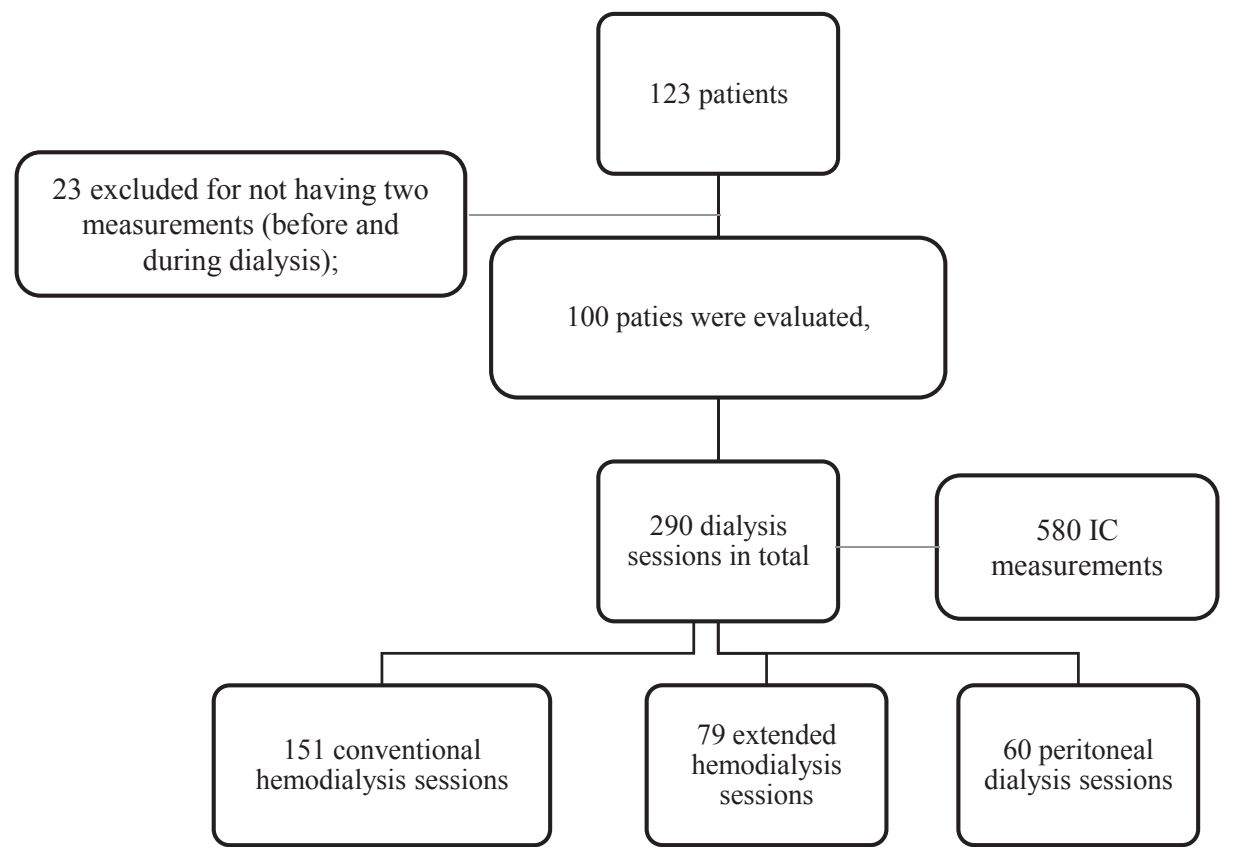

Fig. 1. AKI patients' characterization. 
Table 2

Clinical characteristic of patients undergoing different dialysis modalities.

\begin{tabular}{llll}
\hline Parameter & HVPD & CHD & EHD \\
& $\mathrm{N}=17$ & $\mathrm{~N}=40$ & $\mathrm{~N}=43$ \\
\hline Age (years) & $69 \pm 12.5$ & $57 \pm 18.2^{\mathrm{a}}$ & $60 \pm 16.8$ \\
Male (\%) & $9(53)$ & $29(72.5)$ & $31(72)$ \\
ATN-ISS & $0.64 \pm 0.2$ & $0.62 \pm 0.18$ & $0.67 \pm 0.15$ \\
Weight (Kg) & $71.5 \pm 16.8$ & $82.3 \pm 23.8$ & $74.7 \pm 18.7$ \\
Etiology AKI (\%) & & & \\
$\quad$ Associated with sepsis & $13(76.5)$ & $32(80)$ & $35(81.4)$ \\
Ischemic & $3(17.5)$ & $3(12.5)$ & $4(9.3)$ \\
$\quad$ Nephrotoxic & $1(6)$ & $2(5)$ & $3(7)$ \\
$\quad$ Mixed & $0(0)$ & $1(2.5)$ & $1(2.3)$ \\
Primary diagnosis (\%): & $11(65)$ & $10(25)^{\mathrm{a}}$ & $12(28)^{\mathrm{a}}$ \\
$\quad$ Cardiovascular disease & $4(23)$ & $19(47.5)$ & $23(54)$ \\
$\quad$ Sepsis, severe sepsis, shock & $1(6)$ & $6(15)$ & $1(2)$ \\
$\quad$ Cancer & 0 & $3(7.5)$ & $4(9)$ \\
Liver diseases & $1(6)$ & $2(5)$ & $3(7)$ \\
$\quad$ Trauma & & & \\
Outcome (\%) & $3(17.6)$ & $8(20)$ & $7(16.3)$ \\
$\quad$ Recovery of renal function & $2(11.8)$ & $4(10)$ & $2(4.7)$ \\
$\quad$ Chronic dialysis & $12(71)$ & $28(70)$ & $34(79)$ \\
Death & &
\end{tabular}

AKI - Acute Kidney Injury, ATN-ISS - Acute Tubular Necrosis Individual Score Severity, REE - resting Energy expenditure.

${ }^{\mathrm{a}} \mathrm{p}<0.05$ when compared with HVPD.

\section{Discussion}

The nutritional support goal in critically ill patients with AKI in ICU is to provide enough calories and nutrients to mitigate muscle mass loss and prevent deficiencies, avoiding metabolic disorders exacerbation. Thus, the correct estimative of nutritional requirements is necessary, and the gold standard method for energy expenditure measurement is the IC.

This study aimed to assess whether REE measurement by IC is changed by dialysis, which is a common procedure in patients with severe AKI. Intermittent modalities of hemodialysis (EHD and CHD) and HVPD were evaluated. No significant differences between REE measurement done pre and during dialysis were observed.

Studies conducted in the 70s and 80s are the basis for guidelines that contraindicate IC during dialysis procedure. The rationale is there may be changes in oxygen consumption (VO2) and carbon dioxide production (VCO2) due to acidosis correction, removal of $\mathrm{CO}_{2}$ by dialysate, among other mechanisms, thereby changing the measure of REE $[14,15]$.

The change in VO2 during dialysis is a matter of controversy. VO2 was decreased [19,20], unchanged [21-23] or increased [24-26] during HD and PD [27]. Discrepancies also exist regarding to VCO2 $[21,25,26,28]$. Old studies with ambiguous results turn inaccurate the statement that IC should not be used during dialysis procedure.

A recent study, which evaluated specifically if dialysis procedure alters REE in patients with chronic kidney disease, found an increase in REE during dialysis. This increase still persisted for $2 \mathrm{~h}$ following the end of the procedure (increase 7 and 12\%, respectively, compared with REE pre dialysis). The authors believe that the REE increase may be due, at least in part, increased muscle proteolysis observed during dialysis [29].

Thus, renal replacement therapy (RRT) modality prescribed may impact the loss of amino acids and peptides, and consequently, REE [5,30,31]. Unlike the results of Ikizler et al. [29], this study showed REE was maintained during CHD, EHD and HVPD. This lack of difference between during and pre dialysis times in our study, even though they are catabolic processes, may be due to the fact that during critical illness protein degradation is already markedly increased $[32,33]$. Thus, catabolism is increased even in the patients who are not on dialysis.

Beyond possible alterations in gas exchange, dialysis procedure may affect heat production and removal, as well as blood perfusion, because the procedure alters heat dissipation on skin surface. Furthermore, dialysis could also increase the production of heat. All these factors would contraindicate IC measurement during the procedure. However, few and controversial studies have evaluated the thermal effect of HD on the REE [34,35].

Evaluating REE in hemodialysis with dialysate at different temperatures, Horácek et al. [34] found no change in REE of patients with CKD. On the other hand, Cadena et al. [35] and Rokyta et al. [36] observed changes in REE during dialysis treatment in AKI patients. Cadena et al. [35] observed a REE increase when used dialysate adjusted to maintain body temperature. Rokyta et al. [36] observed decrease in REE during dialysis procedure with "cold" replacement fluid (maintained at $20^{\circ} \mathrm{C}$ ). In our EHD modality, dialysate with low temperature $\left(35.5^{\circ} \mathrm{C}\right)$ is also used. However, this temperature is not as low as in Rokyta et al. study, which may partly explain the lack of difference in REE.

Variables that could alter EE measure, as ventilatory parameters [37,38], use of sedative [39] and body temperature [40], and VAD remained the same during the two measurements in all dialysis modalities. The stability of the evaluated parameters in our patients may explain why REE did not change during dialysis. Modern dialysis procedures aimed minor alterations in respiratory and cardiovascular function in critical patients, so it might not have effect on REE.

This study has some limitations. First, patients in continuous RRT, as continuous hemodiafiltration and hemofiltration

Table 3

Comparisons of ventilatory parameters and drugs between pre-and during dialysis.

\begin{tabular}{|c|c|c|c|c|c|c|}
\hline \multirow[t]{2}{*}{ Time } & \multicolumn{2}{|l|}{ HVPD } & \multicolumn{2}{|l|}{ CHD } & \multicolumn{2}{|l|}{ EHD } \\
\hline & Pre dialysis & During dialysis & Pre dialysis & During dialysis & Pre dialysis & During dialysis \\
\hline REE (kcal) & $2336 \pm 712$ & $2203 \pm 660$ & $2153 \pm 604$ & $2144 \pm 590$ & $2025 \pm 691$ & $1939 \pm 672$ \\
\hline $\mathrm{VT}(\mathrm{ml})$ & $532 \pm 77$ & $515 \pm 99$ & $534 \pm 141$ & $561 \pm 140$ & $521 \pm 118$ & $523 \pm 109$ \\
\hline $\mathrm{Vm}(\mathrm{l} / \mathrm{ml})$ & $8.2 \pm 2.9$ & $8.9 \pm 2.7$ & $9.4 \pm 2.8$ & $10 \pm 3$ & $8 \pm 2.7$ & $8.3 \pm 2.8$ \\
\hline Freq (breath/min) & $16 \pm 4$ & $17 \pm 5$ & $18 \pm 5$ & $19 \pm 6$ & $15 \pm 4$ & $16 \pm 5$ \\
\hline PEEP & $6 \pm 1$ & $6 \pm 1$ & $6 \pm 1$ & $6 \pm 2$ & $6 \pm 2$ & $6 \pm 2$ \\
\hline $\mathrm{FIO}_{2}$ & $38 \pm 10$ & $37 \pm 11$ & $37 \pm 10$ & $37 \pm 10$ & $41 \pm 12$ & $42 \pm 11$ \\
\hline $\operatorname{VAD}(\mu \mathrm{g} / \mathrm{Kg} / \mathrm{min})$ & $0.23(0.18-0.82)^{a}$ & $0.13(0.04-0.75)^{\mathrm{b}}$ & $0.03(0-0.12)$ & $0.03(0-0.15)$ & $0.54(0.4-0.92)^{a}$ & $0.68(0.4-1)^{b}$ \\
\hline Sedative use (\%) & $45(75)$ & $42(70)$ & $88(58)$ & $87(58)$ & $69(87)$ & $70(88)$ \\
\hline Temperature $\left({ }^{\circ} \mathrm{C}\right)$ & $37.5 \pm 1.3$ & $36.9 \pm 0.8$ & $37.6 \pm 0.9$ & $37.5 \pm 0.8$ & $37.9 \pm 0.8$ & $37.9 \pm 0.7$ \\
\hline
\end{tabular}

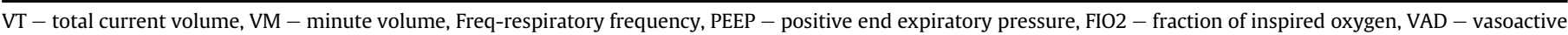
drugs.

a $\mathrm{p}<0.05$ when compared with the time pre dialysis in CHD

b $\mathrm{p}<0.05$ when compared with the time during dialysis of others modalities. 
venovenous were not included, and due to its particular characteristics, these data should not be extrapolated to patients undergoing these modalities. The study was conducted in a single center, which also features a limitation. Nevertheless, we present a consistent sample size, with 290 dialysis sessions evaluated, and to the best of our knowledge this study is the first one evaluating the effect of different dialysis modalities in REE measurement in critically ill patients.

In conclusion, CHD, EHD and HVPD modalities did not change REE. Therefore, IC can be performed during these dialysis procedures. There was no difference between ventilation parameters, sedatives use, body temperature and VAD in both moments. Thus, IC measure contraindication in this modality is not yet proven correct. Therefore, further studies are needed to evaluate REE during continuous RRT, and the real interference in IC measurement, aiming improvement on nutritional advice for critically ill patients with severe AKI.

\section{Author contribution}

Goes CR and Ponce D contributed to the conception and design of the research; Goes CR, Sanches ACS, Balbi AL and Ponce D contributed to the acquisition, analysis, or interpretation of the data; Goes CR, Vogt BP, Ponce D drafted the manuscript; Goes CR, Vogt BP, Balbi AL and Ponce D critically revised the manuscript; and Goes CR, Vogt BP, Sanches ACS, Balbi AL and Ponce D agree to be fully accountable for ensuring the integrity and accuracy of the work. All authors read and approved the final manuscript.

\section{Conflict of interest}

There are no conflicts of interest to disclose.

\section{Acknowledgments}

This study had financial support of grant \#2013/00315-1, São Paulo Research Foundation (FAPESP). Doctorate scholarship was provided to Vogt BP by Coordination of Improvement of Higher Education Personnel (Coordenação de Aperfeiçoamento de Pessoal de Nivel Superior), an organization of the Brazilian federal government under the Ministry of Education.

\section{References}

[1] VA/NIH Acute Renal Failure Trial Network, Palevsky PM, Zhang JH, O'Connor TZ, Chertow GM, Crowley ST, et al. Intensity of renal support in critically ill patients with acute kidney injury. N Engl J Med 2008;3(359):7-20.

[2] Hanna J, Nichol A. Acute renal failure and the critically ill. Anaesth Intensive Care Med 2012;1(13):166-70.

[3] Dvir D, Cohen J, Singer P. Computerized energy balance and complications in critically ill patients: an observational study. Clin Nutr 2006:25:37-44.

[4] Fiaccadori E, Lombardi M, Leonardi S, Rotelli CF, Tortorella G, Borghetti A. Prevalence and clinical outcome associated with preexisting malnutrition in acute renal failure: a prospective cohort study. J Am Soc Nephrol 1999;10:581-93.

[5] McCarthy MS, Phipps SC. Special nutrition challenges: current approach to acute kidney injury. Nutr Clin Pract 2014;29:56-62.

[6] Berbel MN, Góes, R C, Balbi AL, Ponce D. Nutritional parameters are associated with mortality in acute kidney injury. Clinics 2014:69:476-82.

[7] Japur CC, Monteiro JP, Marchini JS, Garcia RWD, Basile-Filho A. Can an adequate energy intake be able to reverse the negative nitrogen balance in mechanically ventilated critically ill patients? J Crit Care 2010;25:445-50.

[8] Heidegger CP, Berger MM, Graf S, Zingg W, Darmon P, Costanza MC, et al. Optimisation of energy provision with supplemental parenteral nutrition in critically ill patients: a randomised controlled clinical trial. Lancet 2013;381:385-93.

[9] Schlein KM, Coulter SP. Best practices for determining resting energy expenditure in critically ill adults. Nutr Clin Pract 2014;29:44-55.

[10] Haugen HA, Chan L-N, Li F. Indirect calorimetry: a practical guide for clinicians. Nutr Clin Pract 2007;22(4):377-88.

[11] Singer P, Singer J. Clinical guide for the use of metabolic carts: indirect calorimetry-no longer the orphan of energy estimation. Nutr Clin Pract 2016;31:30-8
[12] Kolhe NV, Muirhead AW, Wilkes SR, Fluck RJ, Taal MW. National trends in acute kidney injury requiring dialysis in England between 1998 and 2013. Kidney Int 2015;88:1161-9.

[13] Hsu RK, McCulloch CE, Heung M, Saran R, Shahinian VB, Pavkov ME, et al. Exploring potential reasons for the temporal trend in dialysis-requiring AKI in the United States. Clin J Am Soc Nephrol 2016;11:14-20.

[14] Blumberg A, Keller G. Oxygen consumption during maintenance hemodialysis. Nephron 1979;23:276-81.

[15] Mault JR, Dechert RE, Bartlett RH, Swartz RD, Ferguson SK. Oxygen consumption during hemodialysis for acute renal failure. Trans Am Soc Artif Intern Organs 1982;28:510-3.

[16] Kidney Disease: Improving Global Outcomes (KDIGO) Acute Kidney Injury Work Group. KDIGO clinical practice guideline for acute kidney injury. Kidney Int Suppl 2012;2:1-138.

[17] Levey AS, Bosch JP, Lewis JB, Greene T, Rogers N, Roth D. A more accurate method to estimate glomerular filtration rate from serum creatinine: a new prediction equation. Modification of Diet in Renal Disease Study Group. Ann Intern Med 1999;16(130):461-70.

[18] Brito GA, Balbi AL, Abrão JMG, Ponce D. Long-term outcome of patients followed by nephrologists after an acute tubular necrosis episode. Int J Nephrol 2012;2012:361528.

[19] Dolan MJ, Whipp BJ, Davidson WD, Weitzman RE, Wasserman K. Hypopnea associated with acetate hemodialysis: carbon dioxide-flow-dependent ventilation. N Engl J Med 1981;305:72-5.

[20] Faro S, Stabile C, Lopes dos Santos M, Romaldini H, Ratto OR. Central venous blood composition and the pulmonary ventilation during hemodialysis. Nephron 1985:41:45-9.

[21] Carlon GC, Campfield PB, Goldiner PL, Turnbull AD. Hypoxemia during hemodialysis. Crit Care Med 1979;7:497-9.

22] Romaldini H, Rodriguez-Roisin R, Lopez FA, Ziegler TW, Bencowitz HZ, Wagner PD. The mechanisms of arterial hypoxemia during hemodialysis. Am Rev Respir Dis 1984;129:780-4.

[23] Patterson RW, Nissenson AR, Miller J, Smith RT, Narins RG, Sullivan SF. Hypoxemia and pulmonary gas exchange during hemodialysis. J Appl Physiol $1981 ; 50: 259-64$

[24] Hunt JM, Chappell TR, Henrich WL, Rubin LJ. Gas exchange during dialysis Contrasting mechanisms contributing to comparable alterations with acetate and bicarbonate buffers. Am J Med 1984;77:255-60.

[25] Vaziri ND, Wilson A, Mukai D, Darwish R, Rutz A, Hyatt J, et al. Dialysis hypoxemia. Role of dialyzer membrane and dialysate delivery system. Am Med 1984;77:828-33.

[26] Symreng T, Flanigan MJ, Lim VS. Ventilatory and metabolic changes during high efficiency hemodialysis. Kidney Int 1992;41:1064-9.

[27] Fabris A, Biasioli S, Chiaramonte C, Feriani M, Pisani E, Ronco C, et al. Buffer metabolism in continuous ambulatory peritoneal dialysis (CAPD): relationship with respiratory dynamics. Trans Am Soc Artif Intern Organs 1982;28:270-5.

[28] Lim VS, Bier DM, Flanigan MJ, Sum-Ping ST. The effect of hemodialysis on protein metabolism. A leucine kinetic study. J Clin Invest 1993:91:2429-36.

[29] Ikizler TA, Pupim LB, Brouillette JR, Levenhagen DK, Farmer K, Hakim RM, et al. Hemodialysis stimulates muscle and whole body protein loss and alters substrate oxidation. Am J Physiol 2002;282:E107-16.

[30] Umber A, Wolley MJ, Golper TA, Shaver MJ, Marshall MR. Amino acid losses during sustained low efficiency dialysis in critically ill patients with acute kidney injury. Clin Nephrol 2014;81:93-9.

31] Schmidt JJ, Hafer C, Spielmann J, Hadem J, Schönenberger E, Schmidt BMW, et al. Removal characteristics and total dialysate content of glutamine and other amino acids in critically ill patients with acute kidney injury undergoing extended dialysis. Nephron Clin Pract 2014;126:62-6.

[32] Cheatham ML, Safcsak K, Brzezinski SJ, Lube MW. Nitrogen balance, protein loss, and the open abdomen. Crit Care Med 2007;35:127-31.

[33] Rooyackers O, Kouchek-Zadeh R, Tjäder I, Norberg Å, Klaude M, Wernerman J Whole body protein turnover in critically ill patients with multiple organ failure. Clin Nutr 2015;34:95-100.

[34] Horáček J, Sulková SD, Fořtová M, Lopot F, Kalousová M, Sobotka L, et al. Resting energy expenditure and thermal balance during isothermic and thermoneutral haemodialysis - heat production does not explain increased body temperature during haemodialysis. Nephrol Dial Transpl 2007;22:3553-60.

[35] Cadena M, Medel H, Rodrguez F, Flores P, Mariscal A, Franco M, et al. Isothermic vs thermoneutral hemodiafiltration evaluation by indirect calorimetry. Conf Proc A IEEE Eng Med Biol Soc 2008;2008:719-22.

[36] Rokyta R, Matejovic M, Krouzecky A, Opatrny K, Ruzicka J, Novak I. Effects of continuous venovenous haemofiltration-induced cooling on global haemodynamics, splanchnic oxygen and energy balance in critically ill patients. Nephrol Dial Transpl 2004;19:623-30.

[37] Frankenfield DC, Omert LA, Badellino MM, Wiles CE, Bagley SM, Goodarzi S, et al. Correlation between measured energy expenditure and clinically obtained variables in trauma and sepsis patients. J Parenter Enter Nutr 1994;18:398-403.

[38] Faisy C, Guerot E, Diehl J-L, Labrousse J, Fagon J-Y. Assessment of resting energy expenditure in mechanically ventilated patients. Am J Clin Nutr 2003;78:241-9.

[39] Terao Y, Miura K, Saito M, Sekino M, Fukusaki M, Sumikawa K. Ouantitative analysis of the relationship between sedation and resting energy expenditure in postoperative patients. Crit Care Med 2003;31:830-3.

[40] Bruder N, Raynal M, Pellissier D, Courtinat C, François G. Influence of body temperature, with or without sedation, on energy expenditure in severe head-injured patients. Crit Care Med 1998;26:568-72. 